\section{Effects of Relay Selection Strategies on the Spectral Efficiency of Wireless Systems With Half- and Full-Duplex Nodes}

Carlos H. M. de Lima, Member, IEEE, Hirley Alves, Member, IEEE, Pedro. H. J. Nardelli, and Matti Latva-aho, Senior Member, IEEE

\begin{abstract}
This work proposes an analytical framework to study how relay selection strategies perform in half- and full-duplex deployments by combining renewal theory and stochastic geometry. Specifically, we assume that the network nodes-operating in either half- or full-duplex modeare scattered according to a 2-D homogeneous Poisson point process to compute the relay selection cost by using a semi-Markov process. Our results show that: 1) fixed relay outperforms the reactive option in either cases; 2 ) the performance of both reactive and fixed relay strategies depends on the self-interference attenuation in full-duplex scenarios, evincing when they outperform the half-duplex option; and 3) the reactive relay selection suffers from selecting relays at hop basis, while the fixed relay selection benefits most from the full-duplex communication.
\end{abstract}

Index Terms-Full-duplex, relay selection, renewal theory, stochastic geometry.

\section{INTRODUCTION}

I N THE recent years, Full-Duplex (FD) communication has gained considerable attention from both academy and industry [1], [2]. By allowing simultaneous transmission and reception on the same frequency band, FD networks can potentially double the spectral efficiency compared with current Half-Duplex (HD) schemes. However, such potential is harmed by Self-Interference (SI) from the transmit-to-receive antenna [1], [2]. Due to recent advances in the antenna design associated with analog and digital interference cancellation, most of the SIs can be mitigated, and therefore, FD communication becomes feasible and is a step toward meeting high demands of spectral efficiency of $5 \mathrm{G}[3]$.

Current works have shown that FD is a viable solution for small cell deployments [2], due to not only cost and size constraints but also to compatibility with current HD systems. Then, due to the FD capability, the Base Station (BS) can, for instance, simultaneously schedule uplink and Downlink (DL) transmissions (see, for instance, [4]). Moreover, BS can also act as relays for the legacy network as well as for its own subscribed users. Cooperative diversity appeared as a way to combat the effects of the fading, by allowing a single-antenna user to experience spatial diversity [5]. Such schemes perform even better when the relay operates in an FD fashion [6].

Bearing this in mind, and in the context of a dense deployment of small cells, relaying selection becomes an attractive solution given a tradeoff between complexity and efficiency [2], [7]. Relay selection was initially proposed in [7] for HD cooperative networks and has

Manuscript received January 18, 2016; revised August 2, 2016 and November 8, 2016; accepted January 22, 2017. Date of publication January 31, 2017; date of current version August 11,2017. This work was supported by the Strategic Research Council/Aka BCDC Energy under Grant 292854, Aka/SAFE under Grant 303532, and CNPq/Universal program (Brazil). The review of this paper was coordinated by Prof. Y. Gong.

C. H. M. de Lima is with São Paulo State University, 13874-000 São João da Boa Vista, Brazil (e-mail: carlos.lima@ @jbv.unesp.br).

H. Alves, P. H. J. Nardelli, and M. Latva-aho are with the Centre for Wireless Communications, University of Oulu, 90014 Oulu, Finland (e-mail: hirley.alves@oulu.fi; matti.latva-aho@oulu.fi; pedro.nardelli@oulu.fi).

Color versions of one or more of the figures in this paper are available online at http://ieeexplore.ieee.org.

Digital Object Identifier 10.1109/TVT.2017.2661963 gained considerable attention since. Nonetheless, most of the works so far have focused on HD selection algorithms, except for [8] and [9], where the authors focus on the performance of a three-node relaying scenario. For instance, Krikidis et al. [8] focus on selection algorithms for FD amplify-and-forward and model the residual SI at relay as a Rayleigh Random Variable (RV), while, in [9], the authors address the performance of an FD decode-and-forward protocol and assume a simpler residual SI model (constant attenuation factor).

In this work, we assess the performance at the network level of a relay selection procedure for cooperative diversity protocols in the FD mode. To do so, we model the relaying selection procedure as a semi-Markov process, and then, we investigate the impact of the SI and Co-Channel Interference (CCI) on the network performance. Moreover, a dynamic relay selection procedure is considered, where a suitable relay is selected at each hop and the cost of this selection procedure is incorporated into the achievable rate. And yet, we resort to stochastic geometry to model network deployments and capture the dynamics of the network, more specifically the aggregate interference at the receiver, which can be characterized through a cumulant-based framework introduced in [10] and [11].

Our main contributions are summarized as follows.

1) The standard binary tree algorithm is used to implement a relay selection procedure for FD networks.

2) The framework originally introduced in [12] is extended to account for the FD relaying operation.

3) The performance of the aforesaid relay selection protocol is evaluated and compared with its HD counterpart.

4) Different from [8] and [9], our network-level analysis accounts for a more detailed model of the residual SI based on [13] and also characterizes the CCI at the receiver. Actually, instead of modeling the residual SI by a constant value, we consider a more detailed model through a random variate drew from a Ricean distribution with large $K$-factor.

The remainder of this paper is organized as follows. Section II describes the problem under investigation and introduces the system model. Section III describes the network topology and introduces our analytical framework based on the stochastic geometry, while Section IV characterizes the relay selection procedures and the Probability Generating Function (PGF) concept. Section V introduces the semi-Markov process that is used to evaluate the performance of the proposed relay selection mechanism. In Section VI, performance metrics are introduced and then utilized to obtain the numerical results and carry out their discussion. We draw conclusions and final remarks in Section VII.

\section{Problem Description AND SyStem MOdel}

As aforesaid, FD networks constitute a promising alternative to achieve high data rates in the upcoming $5 \mathrm{G}$ systems. In such networks, cooperative nodes benefit most from the inherent rate increase allowed by transmitting and receiving simultaneously. Typically, available literature concentrates on assessing the throughput that is attainable by combining FD and cooperative diversity protocols and overlooks the impact of the underlying relay selection on the final achievable data rate [8].

In this paper, we contribute to previous results by introducing an analytical framework that combines stochastic geometry and semiMarkov processes to account for the cost of selecting a suitable relay. On that regard, we initially use stochastic geometry to derive the distributions of the received power and aggregate interference experienced by the receiver of interest in Section III. Thereafter, we use the PGF concept [14], [15] to characterize the Contention Resolution Interval (CRI) (holding time) of the relay selection algorithms, as can be seen in Sections IV and V. Our investigations extend [12], in which the authors 


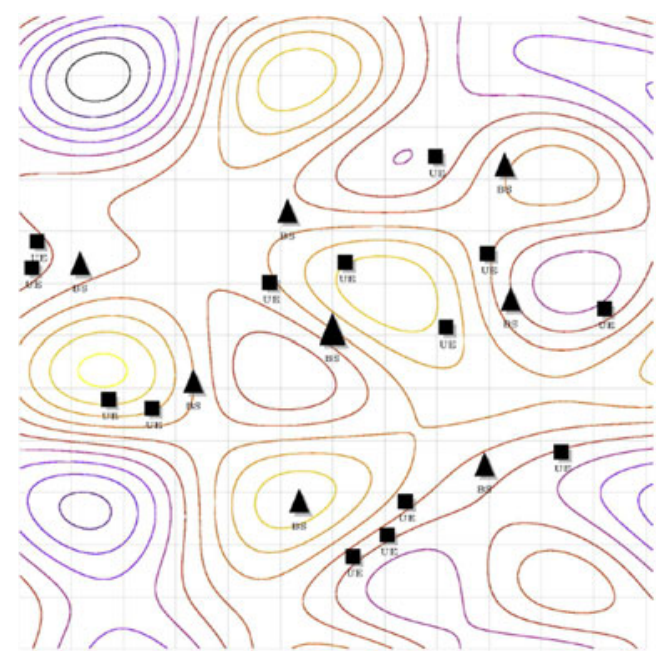

Fig. 1. Illustration of a random (bidimensional) deployment of BSs and user terminals over an arbitrary network area. Shaded squares represent user terminals, while shaded up-triangles depict BSs. A heat map represents the corresponding random composite fading channel, where the fading intensity varies from red/strong to dark blue/weak.

introduce an analytic framework based on semi-Markov processes with constant holding time and apply it to study a 1-D network model.

We assess the performance of interference networks operating in the FD mode under composite fading channel-lognormal shadowing and Nakagami- $m$ fading. The DL of a traditional HD network constitutes our benchmark scenario, wherein the user of interest is interfered by surrounding small cells. BSs independently schedule a random user terminal at every transmission interval. All communicating nodes use antennas with an omnidirectional radiation pattern. BSs and User Equipments (UEs) are also assumed to have full buffer and symmetric traffic patterns [16].

Consider that all channels are quasi-static and undergo a composite fading distribution [17], which is approximated by a lognormal distribution with mean and variance (in logarithmic scale) given by $\mu_{\mathrm{dB}}=\xi[\psi(m)-\ln (m)]+\mu_{\Omega_{p}}$ and $\sigma_{\mathrm{dB}}^{2}=\xi^{2} \zeta(2, m)+\sigma_{\Omega_{p}}^{2}$, where $\psi(m)$ is the Euler psi function, and $\zeta(2, m)$ is the generalized Riemann zeta function [18]. Radio links are affected by path loss, large-scale shadowing, and multipath fading, which are assumed to be mutually independent and multiplicative phenomena [19]. The received power at the user of interest $u_{0}$ from an arbitrary transmitter $b_{i}$ located $r_{i 0}$ meters away is $Y_{i 0}=p_{i 0} r_{i 0}^{-\alpha} x_{i 0}$, where $p_{i 0}$ yields the transmit power, $\alpha$ is the path-loss exponent, and $x_{i 0}$ represents the composite fading channel. In what follows, when there is no fear of ambiguity, let us omit the subscripts of the interference component terms. Fig. 1 illustrates a realization of the random network topology, where UEs and small cell BSs are uniformly scattered over network area.

\section{NeTwORK DEPLOYMENT AND STOCHASTIC GEOMETRY}

Active nodes constitute a homogeneous Poisson Point Processes (PPPs) $\Phi$ with intensity $\lambda$ in $\mathbb{R}^{2}$. The number of nodes in an arbitrary region $\mathcal{R}$ of area $A$ is a Poisson RV with parameter $\lambda A$ [20].

The fading effect is assumed as a random mark associated with each point of $\Phi$; thus, $\widetilde{\Phi}=\{(\varphi, x) ; \varphi \in \Phi\}$ corresponds to a marked point process on the product space $\mathbb{R}^{2} \times \mathbb{R}^{+}$, whose random points $\varphi$ denoting transmitter locations belong to $\Phi$ [20], [21]. The network deployment model is given by a spatial PPPss $\Phi^{\mathrm{BS}}\left(\Phi^{\mathrm{UE}}\right)$, whose random points $\varphi$ represent the locations of BSs (UEs). In addition, we consider a fixed number of potential relays that detect source's message and take part in the selection procedure; it is well known that such conditional distribution of points within an arbitrary forwarding region $A$ is described by a binomial point process [21]. As shown next, by employing stochastic geometry concepts to this deployment model, it is possible to conveniently derive closed-form expressions to characterize the distributions of the received power and corresponding aggregate $\mathrm{CCI}$ with respect to the receiver of interest. Indeed, the path-loss attenuation (function of the distance to the tagged user), the lognormal shadowing, and Nakagami- $m$ fading are combined using the framework of [10] and [22] to obtain the distribution of the received power at the user of interest.

\section{A. Received Power From a Random Transmitter}

We resort to the framework introduced in [10] and [11] to attain the characteristic function and the respective cumulants $(\kappa)$ of the received power and aggregate interference of random transmitters within the forwarding region of the user of interest. Consider an RV $Y=R^{-\alpha} X$ describing the power received at the tagged receiver from a random transmitter in $\mathcal{O}$ (as defined in Section II), with $R$ varying from $R_{m}$ to $R_{M}$, and $X \sim \operatorname{Lognormal}\left(\mu_{\mathrm{dB}}, \sigma_{\mathrm{dB}}\right)$ representing the squared envelope of the composite fading channel. Then, the characteristic function of $Y$ is [11, Appendix B] $\Psi_{Y}(\omega)=2\left(R_{M}^{2}-R_{m}^{2}\right)^{-1} \mathrm{E}_{X}[\mathrm{R}(\omega)]$, where $\mathrm{R}(\omega)=\int_{R_{m}}^{R_{M}} \exp \left(j \omega p r^{-\alpha} x\right) r d r$, and $\mathrm{E}_{X}[\cdot]$ yields the expectation of the enclosed expression over the $\mathrm{RV} X$ and is a general formulation, which characterizes the distribution of any random transmitter within the reception range of a tagged receiver. Thereafter, the $n$th cumulant of $Y$ is given by [11, Appendix C]

$$
\kappa_{n}=\frac{1}{\imath^{n}} \sum_{k=0}^{n} g^{(k)}\left(\beta_{0}\right) \cdot B_{n, k}\left[\beta_{1}, \beta_{2}, \ldots, \beta_{(n-k+1)}\right]
$$

where $\imath$ yields the imaginary unity, $g(u)=\ln (u), \quad B_{n, k}$ $\left[\beta_{1}, \beta_{2}, \ldots, \beta_{(n-k+1)}\right]$ is the partial Bell polynomial [23], and $\beta_{n}=$ $\imath^{n} p^{n} \times \frac{R_{m}^{2-n \alpha}-R_{M}^{2-n \alpha}}{n \alpha-2} \mathrm{E}_{X}\left[x^{n}\right]$.

Next, we use the aforesaid cumulant-based framework to characterize the aggregate interference at the user of interest for both the HD and FD configuration scenarios. Under the assumptions of Section II and with respect to the tagged receiver, we now use our analytical framework to characterize the resulting aggregate CCI in two Evaluation Scenarios (ESs): ES1 is the benchmark scenario where BSs and UEs operate in the HD mode, while in ES2, both BSs and UEs operate in the FD mode.

\section{B. Aggregate CCI From a Poisson Field of Interferers}

In our benchmark scenario, denoted HD relaying, all nodes operate in the $\mathrm{HD}$ mode. The corresponding aggregate $\mathrm{CCI}$ at a tagged receiver is then given by $Z_{0}^{\mathrm{HD}}=\sum_{\left(\varphi_{i}, x_{i}\right) \in \widetilde{\Phi}^{\mathrm{BS}}} Y_{i 0}$. Notice that the tagged receiver only experiences interference coming from nearby BSs, since we assume the DL as the benchmark scenario.

In this context, the aggregate $\mathrm{CCI}$ at the tagged receiver is [10, Section V]

$$
\kappa_{n}\left(\widetilde{\Phi}^{\mathrm{BS}}\right)=\frac{2 \pi \lambda p^{n}}{n \alpha-2}\left(R_{m}^{2-\alpha n}-R_{M}^{2-\alpha n}\right) \mathrm{E}_{X}^{n}[0, \infty] .
$$

\section{Aggregate CCI in the FD Configuration}

In the FD configuration, the tagged receiver is subject to the interference from FD serving BSs, other FD UEs, and its intrinsic SI component. The SI channel between transmitting and receiving antennas of an FD transceiver exhibits a strong line-of-sight component and can be represented using a Ricean distribution with a large $K$-factor [24] (a $K$-factor of $14.8 \mathrm{~dB}$ is used, which corresponds to a Nakagami distribution with the parameter $m=16$ ). As a result, the aggregate $\mathrm{CCI}$ at the tagged receiver is characterized by

$$
Z_{0}^{\mathrm{FD}}=\delta p_{00} x_{00}+\sum_{\left(\varphi_{i}, x_{i}\right) \in \widetilde{\Phi}_{\mathrm{BS}}} Y_{i 0}+\sum_{\left(\varphi_{j}, x_{j}\right) \in \widetilde{\Phi} \mathrm{UE}} Y_{j 0}
$$


where $p_{00}$ and $\delta$ represent the SI component and the corresponding attenuation factor, respectively.

Since BSs and UEs are assumed to be independently scattered over the network deployment area, the resulting processes $\left(\Phi^{\mathrm{BS}}\right.$ and $\Phi^{\mathrm{UE}}$, respectively) from each such tier are also independent [20]. Therefore, the cumulant additivity property is employed to obtain the aggregate CCI from multiple interfering tiers [10]. In other words, if $U$ and $V$ are independent RVs, then we can derive the cumulant of their sum as $\kappa_{n}(U+V)=\kappa_{n}(U)+\kappa_{n}(V)$.

\section{Signal-to-Interference Ratio and Outage Probability}

By using the results from previous sections, it is straightforward to compute the Signal-to-Interference Ratio (SIR) and the outage probability of the ESs under study. The SIR and the outage probability are used to assess how the relay selection strategies perform in HD and FD configuration modes as follows.

Proposition 1: Let $V_{0}$ and $V$ be normal RVs (in logarithmic scale) representing the power received from the desired transmitter and the $\mathrm{CCI}$ at the receiver of interest. Under the assumption of the composite fading with the Gamma-lognormal distribution, the SIR at the tagged receiver is $\Gamma \sim \operatorname{Normal}\left(\mu_{V_{0}}-\mu_{V}, \sigma_{V_{0}}^{2}+\sigma_{V}^{2}\right)$, and the outage probability is given by $\operatorname{Pr}\left[\Gamma<\gamma_{\mathrm{th}}\right]=\mathrm{Q}\left[\left(\mu_{\Gamma}-\gamma_{\mathrm{th}}\right) / \sigma_{\Gamma}\right]$, where $\mu_{\Gamma}=$ $\mu_{V_{0}}-\mu_{V}$ and $\sigma_{\Gamma}^{2}=\sigma_{V_{0}}^{2}+\sigma_{V}^{2}$. Notice that the lognormal parameters can be attained as follows: $\mu=\ln \left(\kappa_{1}^{2} / \sqrt{\kappa_{1}^{2}+\kappa_{2}}\right)$, and $\sigma^{2}=$ $\ln \left(1+\kappa_{2} / \kappa_{1}^{2}\right)$, where $\kappa_{1}$ and $\kappa_{2}$ are given by (1).

Proof: The SIR distribution is given by the quotient of two independent lognormal RVs, namely, $e^{V_{0}}$ which is the received power from the target transmitter, and $e^{V}$ which is an equivalent lognormal $\mathrm{RV}$ approximating the aggregate $\mathrm{CCI}$ at the tagged receiver [25].

\section{Relay SElection Algorithm AND PRobability GENERATING FUNCTION}

Hereafter, we characterize the cost of selecting a suitable relay at hop basis on the achievable throughput. To do that, we first account for the relay selection overhead using the PGF of the CRI and later combine the impact of the network characteristics with the relay selection mechanism throughout a semi-Markov process. In this work, a totally random approach based solely on the standard tree algorithm is used to implement the relay selection algorithm [26]. It is worth noticing that we use the conditional PGF of the CRI length $\left(L_{N}\right)$ that is derived in [14] and [15] for the same standard tree algorithm under investigation. Next, due to space limitations, we only summarize the main results regarding the relay selection procedure.

\section{A. Relay Selection Algorithm}

Two relay selection approaches are considered in this work: 1) a static one, whereby a relay node is preassigned to forward source packets; and 2) a reactive strategy, by which a relay node is identified based on the selection criteria-i.e., the neighboring node that provides the longest advancement toward the final destination at each hop. While the former configuration may be more applicable to legacy networks where a small cell may provide the last hop to cell edge users, the latter is tailored to upcoming $5 \mathrm{G}$ networks, where humans and machines communicate in large-scale deployment scenarios [27]. On one hand, the static relay procedure relies on long-term characteristics of the deployment scenarios, i.e., the relay is selected $a$ priori following a network planning (it is arbitrary in our investigations) and does not change. On the other hand, the reactive relay procedure selects a random (new) relay within the transmission range every time a packet needs to be forwarded to the destination [12], [14], [28].

Succinctly, the reactive relay selection algorithm is implemented through a carrier sense collision avoidance mechanism similar to the IEEE 802.11 handshake using Request to Send (RTS)/Clear To Send

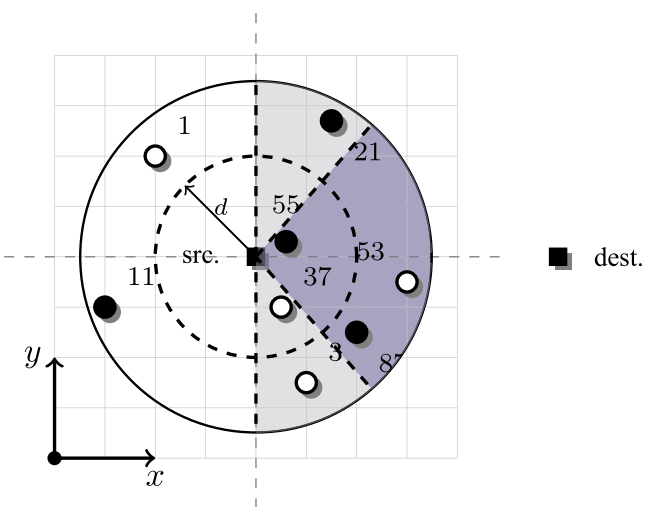

Fig. 2. Illustration of the sectoral decision region with $Q=2$ splitting groups and angular aperture of $180^{\circ}$. Dashed lines defined the forwarding region (shaded regions in light gray and blue), black circles identify awake nodes, and white circles identify asleep nodes. All awake neighbors within the shaded region are eligible relays.

(CTS) messages. The source transmits an RTS packet to initiate the relay selection procedure. Nodes that listen to this request reply with a CTS packet based on the predetermined probability of accessing the channel, $p$. If a collision occurs, nodes that have transmitted in previous slot retransmit or not based on a random access process similar to a $Q$-sided coin. To select the best suitable relay (greed criteria), i.e., the closest node to the destination whether there is one available, the source node should receive the replies from all the candidate relays [14], [15]. We consider the conditional CRI length when $N$ nodes initially collide (which corresponds to a binomial point process [21]). Here, aiming at shorten the selection procedure, ${ }^{1}$ the first node (at a random location within transmission range) to successfully reply to the source is selected as the next hop relay-note that this mechanism differs from the one used in [14] and [15], which waits for the replies of all neighboring relay candidates in order to select the most suitable based on the predefined selection criteria.

We use the relay selection mechanism introduced in [14] and [15], which combines the standard tree algorithm to resolve contentions and sectoral decision regions to identify the potential relays. Fig. 2 illustrates a snapshot of the sectoral decision region that is used in conjunction with the standard tree-based solution. In the next section, the PGF is used to characterize the distribution of the CRI associated with the selection of a random relay at hop basis.

Remark 1: Notice that current 802.11 standard implements the RTS/CTS handshake as an optional feature to avoid frames collisions; besides, more recent standards such as 802.22 and 802.11af also incorporate cognitive radio functionalities, which makes it even easier to deal with side information [14].

Remark 2: In long-term evolution (LTE) systems, the contentionbased random channel is based on ALOHA-type communication and is susceptible to performance degradation due to a high collision probability in the transmission of the preambles in dense deployments. Indeed, the contention-based operation of the LTE physical random access channel is also established by the signaling exchange of messages, namely, preamble transmission, random access response, connection request, and contention resolution [27], [29]

\section{B. PGF of the Relay Selection Algorithm}

The conditional CRI length considering a $Q$-sided fair coin is generalized as

$$
L_{N}= \begin{cases}1, & \text { if } N=0,1 \\ 1+\sum_{j=1}^{Q} L_{I_{j}}, & \text { if } N \geq 2\end{cases}
$$

${ }^{1}$ At the cost of selecting a relay that is not necessarily the closest one to the final destination. 
where $I_{j}$ is a discrete RV describing the number of candidate relays that tossed the $j$ value of the $Q$-side coin. For a binary splitting tree, the PGF of the time spent by a random node in the relay selection procedure is given by the following proposition.

Proposition 2: Under the assumptions of a binary standard tree algorithm (unbiased coin) and $N$ conflicting nodes, the PGF of the CRI for a tagged packet is

$$
\begin{aligned}
& G_{N+1}(z)=\frac{1}{2}\left[G_{N+1}^{(0)}(z)+G_{N+1}^{(1)}(z)\right] \text { with } \\
& G_{N+1}^{(0)}(z)=z \sum_{k=0}^{N} B_{N, k} G_{k+1}(z) \text { and } \\
& G_{N+1}^{(1)}(z)=z \sum_{k=0}^{N} B_{N, k} Q_{k}(z) G_{N-k+1}(z)
\end{aligned}
$$

where $G_{1}=z, G_{N+1}^{(0)}(z)$ accounts for the CRI when the tagged node joins the first subset (flipped 0$), G_{N+1}^{(1)}(z)$ describes the resolution interval when the tagged node joins the second subset (flipped 1), and $B_{N, k}=\operatorname{Pr}[k$ flipped $0 \mid N$ contending nodes $]$. Note that the tagged packet may be in each subset with the equal probability.

Proof: Let $N>1$ be the number of contending nodes that start the CRI. For a fair binary splinting tree, we have $B_{N, k}=\left(\begin{array}{l}N \\ k\end{array}\right) 2^{-k}$. Thus, if the first splitting group has $k$ members, the second contains the remaining $N-k$ nodes. Each such sub-CRI is statically indistinguishable from a general CRI with equal number of elements. Therefore, the recursive formula $Q_{N}(z)=\sum_{k=0}^{N} B_{N, k} Q_{k}(z) Q_{n-k}(z)$ is obtained. By the same line of reasoning, we can define

$$
\begin{aligned}
G_{N+1}(z) & =\operatorname{Pr}\left[t_{0}=k \mid N \text { other nodes collide }\right] z^{k} \\
G_{N+1}^{(s)}(z) & =\operatorname{Pr}\left[t_{0}=k \mid N\right. \text { other nodes collide } \\
& \text { and the tagged node flips } s] z^{k}
\end{aligned}
$$

where $t_{0}$ yields the time a tagged packet spends in the CRI before being successfully transmitted. Finally, computing the corresponding probabilities for a fair binary spiting tree, we obtain (5).

In other words, Proposition 2 gives the time a tagged packet takes to be successfully transmitted. When a random node within source's transmission range is selected, its distance distribution is characterized by (1). To determine the holding time matrix, we resort to the factorial moment concept so as to compute the mean and variance of CRI for a tagged packet from (2).

Definition 1: Let $X$ be a discrete RV taking nonnegative integer values; then, the $k$ th factorial moment of $X$ is given as $\mathrm{E}[X(X-1) \cdots(X-k+1)]=G^{(k)}\left(1^{-}\right), k \geq 0$ [30].

Then, we obtain the following results from Definition 1: $G(1)=$ $\sum_{k} \operatorname{Pr}[X=k], G^{\prime}(1)=\mathrm{E}[X]$ and $G^{\prime \prime}(1)=\mathrm{E}[X(X-1)]$.

\section{SEMI-MARKov PROCESS}

Markov processes permit random systems whose outcome at any given instant depends only on the outcome that proceeds it (memoryless property; if the present is specified, the past has no influence in the future). A Markov chain is a special kind of Markov process, wherein the future evolution of the process depends on the present state and not on how it arrived at that state [31]. Typically, such Markov models have the property that a transition occurs at every instant-even if the transition returns to the previous occupied state, it occurs anyway. In this work, we use a semi-Markov process that is associated with the embedded Markov chain in Fig. 3 that tracks the relay selection protocols states. The semi-Markov process theory allow us to obtain the steady-state average delay and throughput during a cycle taking any state of the chain as a reference [30], [31]. Hence, at transition instants, the semi-Markov process behaves just as a Markov process. It is

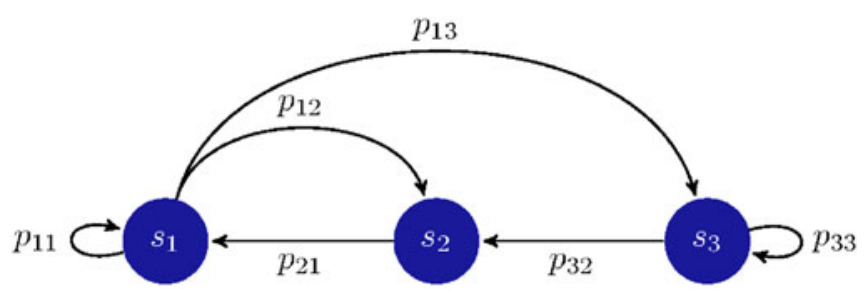

Fig. 3. Illustration of a Semi-Markov process with three states $s_{i}, \quad i \in$ $\{1,2,3\} . s_{1}$ represents transmission, $s_{2}$ relay, and $s_{3}$ retransmission.

used to combine the network topology (including radio channel) that is captured by the stochastic geometry with the routing protocol dynamics that are captured by the PGF framework. With the semi-Markov process, the elapsed time between transitions may take several unit intervals, while, at transition instants, it behaves as a typical Markov process [30]. The semi-Markov process depends on the transition that occurred, though the transition instants follow a distinct probabilistic mechanism [30]. Throughout our investigation, we consider the states presented in Fig. 3: $s_{1}, s_{2}$, and $s_{3}$ correspond to the transmission, relay and retransmission states, respectively. The corresponding state transition matrix is given by

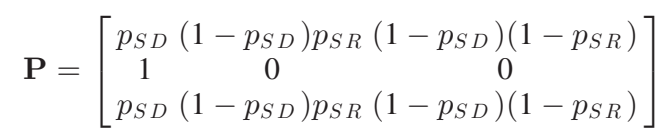

where each element $p_{i j}$ yields the transition probability from state $i$ to $j$, and $p_{S D}$ and $p_{S R}$ yield the probability that the source message is correctly received by the final destination and relay node, respectively.

Remark 3: The Markov process model has the property that a transition is made at every time instant. Differently, the semi Markov model is a more general class of processes, where the elapsed time between transitions may take several unit time intervals. Then, it brings much more flexibility to the problem of modeling dynamic probabilistic systems. In fact, the successive state occupancies are governed by the transitions probabilities of an embedded Markov process, while the holding time in each state follows an integer-valued random distribution-the conflict resolution interval, see (4) in Section IV-which depends on both the current and the next states [30].

The PGF of the CRI $G_{N}(z)$ characterizes the relay selection overhead at each hop. The probability that a certain CRI takes $k$ slots can be retrieved from the respective PGF using the following proposition.

Proposition 3: The probability mass function of $L_{N}$, the length of the CRI (in transmission slots) conditional on the number of contending nodes, is $\operatorname{Pr}[X=x]=G^{(x)}(0) / x$ !

Proof: From the PGF definition, it is straightforward that

$$
G_{N}(z)=\mathrm{E}\left[z^{X}\right]=\sum_{x=0}^{\infty} \operatorname{Pr}[X=x] z^{x} .
$$

Then, taking the $n$th derivative of (11) and making $z=0$, we attain the result in Proposition 3.

The holding time matrix incorporates into the embedded Markov chain of Fig. 3 the time spent in a given state between successive transitions

$$
\mathbf{H}=\left[\begin{array}{ccc}
1 & 1+L_{N} & 1+L_{N} \\
1 & 1 & 1 \\
1 & 1+L_{N} & 1+L_{N}
\end{array}\right]
$$

where each element $h_{i j}$ yields the time elapsed in state $i$ before transition to $j$.

\section{A. Steady-State Throughput Efficiency}

Let $i$ be an arbitrary state chosen as reference, and $\pi_{i}$ be its steadystate probability, where the vector $\pi$ corresponds to the steady-state 


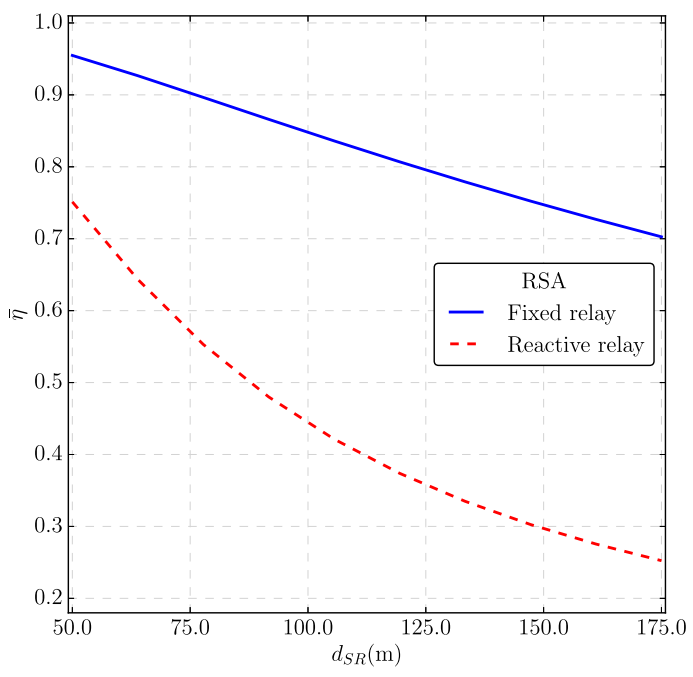

Fig. 4. Steady-state throughput efficiency for increasing separation distance between source-destination pairs in the HD configuration.

distribution of the embedded Markov chain (see Fig. 3). The time delay associated with the transition from state $i$ to $j$ is given by $D_{i j}$. Similarly, $R_{i j}$ is the reward associated with the transition from state $i$ to $j$. A cycle is defined as the time between two consecutive passages for the reference state states. A message is successfully delivered to the destination node when the process returns to the state $s_{1}$. The reward function $\mathrm{R}(\tau)$ corresponds to the cumulative reward after a period $\tau$ and yields the total amount of correct receptions. According to the fundamental renewal-reward theorem [30], the long-term throughput is given by the following value, It is worth noticing that the resulting steady-state throughput efficiency is independent of the choice of the reference state. Moreover, the long-term evaluation of $\mathrm{R}(\tau) / \tau$ actually corresponds to the normalized throughput of the protocol, as introduced in [32] and revisited in [28]

$$
\eta=\lim _{\tau \rightarrow \infty} \frac{\mathrm{R}(\tau)}{\tau}=\frac{\sum_{i=1}^{K} \pi_{i} \overline{\mathrm{R}}_{i}}{\sum_{i=1}^{K} \pi_{i} \overline{\mathrm{D}}_{i}}
$$

where $K$ yields the number of states, $\overline{\mathrm{R}}=\sum_{j}^{K} p_{i j} \mathrm{R}_{i j}$ is the mean rewarding, while $\overline{\mathrm{H}}=\sum_{j}^{K} p_{i j} \mathrm{~h}_{i j}$ is the mean waiting time.

\section{NUMERICAL RESULTS}

For both desired link and interferers, a composite fading channel model with a Nakagami- $m$ parameter of $m=16$ (which corresponds to a Ricean fading channel with factor $K=14.8 \mathrm{~dB}$ ) and a shadowing standard deviation of $10 \mathrm{~dB}$ is considered, as well as channel reciprocity between communication pairs. Interferes are scattered over the network area with $R_{m}=25$ and $R_{M}=500 \mathrm{~m}$, while $\lambda=5 \times 10^{-5}$ nodes $/ \mathrm{m}^{2}$ is considered (which corresponds to nearly 40 interferers on average), and the path-loss attenuation is set to 3 . The CRI is conditioned on the mean number of candidate relays on the forward region of the source node. Without loss of generality, we consider three contending relays within source's forwarding region to compute the relay selection interval. We assume that data and control information occupies one transmission interval.

In an HD configuration, Fig. 4 shows the steady-state throughput efficiency $\bar{\eta}$ for increasing separation distance between source-destination pairs. As evidenced by the reactive relay curve (red-dashed line), the steady-state throughput is severely compromised by the relay selection. On the other hand, when a fixed relay is considered within source's transmission range, $\bar{\eta}$ becomes much less susceptible to the degrading effect of a longer separation distance between the source and the destination.

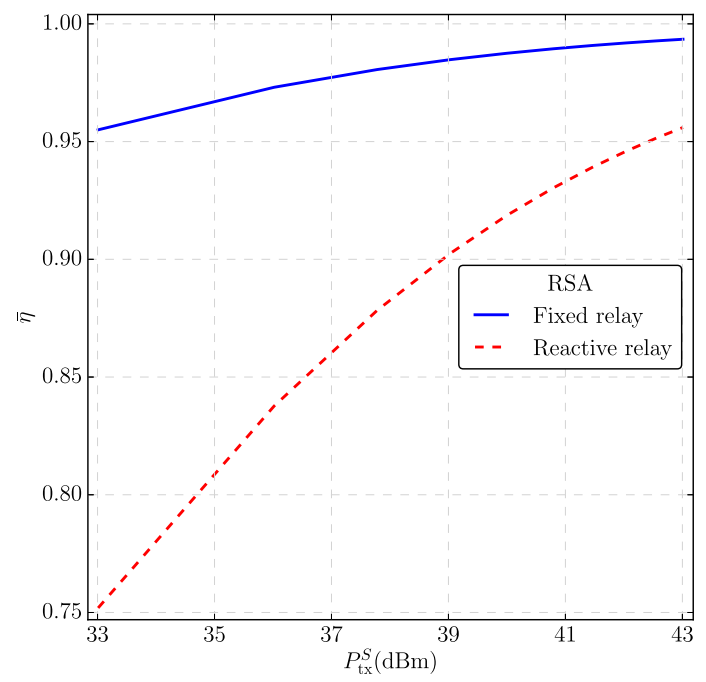

Fig. 5. Steady-state throughput efficiency increasing source's transmission power in the HD configuration.

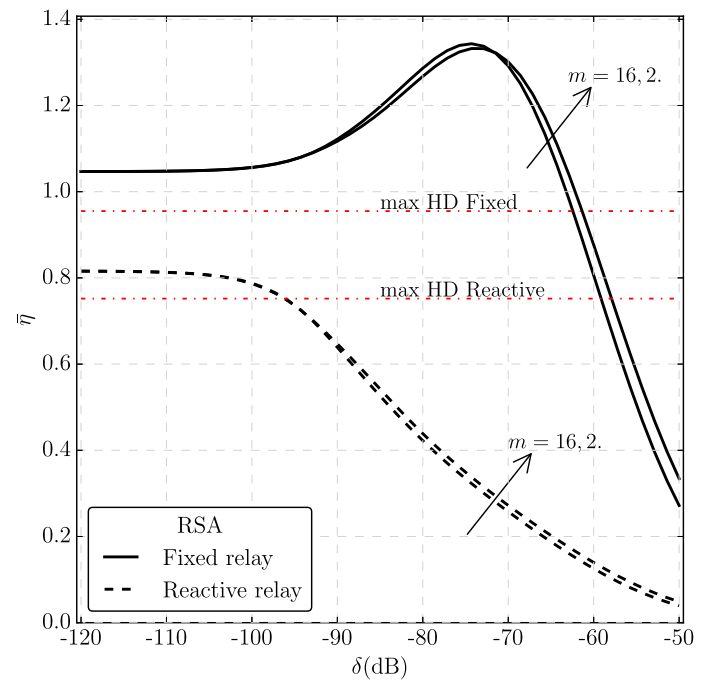

Fig. 6. Steady-state throughput efficiency for decreasing SI attenuation in the FD configuration.

Fig. 5 presents the steady-state throughput for increasing transmission power of the source node, whereas interferers transmit at $30 \mathrm{dBm}$. The separation distance between the source and the destination is kept at $50 \mathrm{~m}$, and the relay is randomly located in the source's forwarding region. As expected, the steady-state throughput improves with higher source's transmission power. The reactive relay benefits mainly from high transmission power because a source can reach the destination more often without undergoing long relay selection intervals to select a suitable relay.

The steady-state throughput efficiency for decreasing SI attenuation with the FD configuration is shown in Fig. 6. The separation distance between the source and the destination is set to $50 \mathrm{~m}$. As previously observed in the HD configuration, the steady-state throughput with a reactive relay is severely degraded by the relay selection procedure and barely outperforms the HD scenario with 100-dB SI attenuation. It is also shown how the steady-state throughput efficiency scales for $m=2$ and 16 . As can be seen, $\bar{\eta}$ slightly degrades when the Nakagami- $m$ parameter of the SI component increases from 2 (nonline of sight) to 16 (line of sight). 
Regarding the fixed relay scheme, the FD configuration with SI attenuation ranging from 100 to nearly $70 \mathrm{~dB}$ shows a much better performance than the HD mode, whereas the performance degrades faster with lower attenuation values. From 120 to $100 \mathrm{~dB}$, the FD gain is negligible, because the SI is low and the source node reaches the destination directly. However, for lower SI attenuation, the source node uses the relay more often, which increases $\bar{\eta}$ (the reward doubles). Despite that, if the attenuation is lower than $70 \mathrm{~dB}$, the SI also compromises the relay performance and degrades the steady-state throughput efficiency considerably. Moreover, as indicated by the dotted-dashed threshold lines, the FD configuration outperforms the HD one for high SI attenuation values. However, if the SI attenuation drops below $70 \mathrm{~dB}$, the FD performance degrades and HD becomes a better alternative. In fact, it would be advantageous to consider a dynamic forwarding scheme, by which potential relays would change between FD and HD depending on the attainable SI value.

\section{CONCLUSION AND FINAL REMARKS}

In this work, we assess how in-band FD relaying performs for distinct selection strategies, namely fixed and reactive. Our investigations are carried out using an analytical framework based on the stochastic geometry and the semi-Markov process. The former is used to model the network deployment area and radio channel, while the latter characterizes the cost of the relay selection procedure under study. Our results show that the benefits of the FD configuration are compromised by the frequent selection of the next hop relay. Moreover, the steadystate throughput degrades depending on the relative distance between the source, the relay, and the destination, as well as the respective SI attenuation. As pointed out in [14] and [15], a binary search tree implementation does not take full advantage of the side information (local network topology) to shorten the relay selection time. Hence, we plan to extend this work by considering Dutch auction-based relay selection to exploit the local topology of the network in order to shorten the selection transactions. We also expect to assess the secrecy of the network, as in [33], by employing a similar framework.

\section{REFERENCES}

[1] A. Sabharwal, P. Schniter, D. Guo, D. Bliss, S. Rangarajan, and R. Wichman, "In-band full-duplex wireless: Challenges and opportunities," IEEE J. Sel. Areas Commun., vol. 32, no. 9, pp. 1637-1652, Sep. 2014.

[2] G. Liu, F. Yu, H. Ji, V. Leung, and X. Li, "In-band full-duplex relaying: A survey, research issues and challenges," IEEE Commun. Surveys Tuts., vol. 17, no. 2, pp. 500-524, Second Quarter 2015.

[3] S. Hong et al., "Applications of self-interference cancellation in $5 \mathrm{G}$ and beyond," IEEE Commun. Mag., vol. 52, no. 2, pp. 114-121, Feb. 2014.

[4] C. H. M. de Lima, H. Alves, P. H. J. Nardelli, and M. Latva-aho, "Hybrid half- and full-duplex communications under correlated lognormal shadowing," in Proc. IEEE 81st Veh. Technol. Conf., Glasgow, U.K., May 2015, pp. 1-5.

[5] F. Gomez-Cuba, R. Asorey-Cacheda, and F. J. Gonzalez-Castano, "A survey on cooperative diversity for wireless networks," IEEE Commun. Surveys Tuts., vol. 14, no. 3, pp. 822-835, Third Quarter 2012.

[6] H. Alves, D. B. da Costa, R. D. Souza, and M. Latva-aho, "Performance of block-Markov full duplex relaying with self interference in Nakagamim fading," IEEE Trans. Wireless Commun., vol. 2, no. 3, pp. 311-314, Jun. 2013.

[7] A. Bletsas, A. Khisti, D. Reed, and A. Lippman, "A simple cooperative diversity method based on network path selection," IEEE J. Sel. Areas Commun., vol. 24, no. 3, pp. 659-672, Mar. 2006.

[8] I. Krikidis, H. Suraweera, P. Smith, and C. Yuen, "Full-duplex relay selection for amplify-and-forward cooperative networks," IEEE Trans. Wireless Commun., vol. 11, no. 12, pp. 4381-4393, Dec. 2012.

[9] X. Rui, J. Hou, and L. Zhou, "On the performance of full-duplex relaying with relay selection," Electron. Lett., vol. 46, no. 25, pp. 1674-1676, 2010.

[10] C. H. M. de Lima, M. Bennis, and M. Latva-aho, "Coordination mechanisms for self-organizing femtocells in two-tier coexistence scenarios," IEEE Trans. Wireless Commun., vol. 11, no. 6, pp. 2212-2223, Jun. 2012.
[11] C. H. M. de Lima, M. Bennis, and M. Latva-aho, "Statistical analysis of self-organizing heterogeneous networks with biased cell association and interference avoidance," IEEE Trans. Veh. Technol., vol. 62, no. 5, pp. 1950-1961, Jun. 2013 (Special Section on Self-Organizing Radio Networks).

[12] N. Marchenko and C. Bettstetter, "Impact of relay selection overhead in cooperative diversity protocols," in Proc. IEEE Veh. Technol. Conf., Sep. 2011, pp. 1-4.

[13] M. Duarte and A. Sabharwal, "Full-duplex wireless communications using off-the-shelf radios: Feasibility and first results," in Proc. 44th Asilomar Conf., 2010, pp. 1558-1562.

[14] C. H. M. de Lima and G. T. F. de Abreu, "Analysis of contention-based relay selection mechanisms in autonomous multi-hop networks," in Proc. IEEE Inf. Theory Workshop Netw. Inf. Theory, Volos, Greece, Jun. 2009, pp. 186-190.

[15] C. Lima, P. Nardelli, H. Alves, and M. Latva-Aho, "Contention-based geographic forwarding strategies for wireless sensors networks," IEEE Sens. J., vol. 16, no. 7, pp. 2186-2195, Apr. 2015.

[16] D. Bertsekas and R. Gallager, Data Networks, 2nd ed. Englewood Cliffs, NJ, USA: Prentice-Hall, 1992.

[17] G. L. Stüber, Principles of Mobile Communication, 3rd ed. New York, NY, USA: Springer, 2011.

[18] M. Abramowitz and I. A. Stegun, Handbook of Mathematical Functions with Formulas, Graphs, and Mathematical Tables, 9th ed. New York, NY, USA: Dover, 1965.

[19] J. Ilow and D. Hatzinakos, "Analytic alpha-stable noise modeling in a poisson field of interferers or scatterers," IEEE Trans. Signal Process., vol. 46, no. 6, pp. 1601-1611, Jun. 1998.

[20] J. F. C. Kingman, Poisson Processes. Oxford, U.K.: Oxford Univ., 1993.

[21] A. Baddeley, I. Bárány, R. Schneider, and W. Weil, Stochastic Geometry. New York, NY, USA: Springer, 2006.

[22] A. Ghasemi and E. S. Sousa, "Interference aggregation in spectrumsensing cognitive wireless networks," IEEE J. Sel. Areas Commun. vol. 2, no. 1, pp. 41-56, Feb. 2008.

[23] E. T. Bell, "Exponential polynomials," Ann. Math., vol. 35, no. 2, pp. 258-277, Apr. 1934

[24] M. Duarte, C. Dick, and A. Sabharwal, "Experiment-driven characterization of full-duplex wireless systems," IEEE Trans. Wireless Commun., vol. 11, no. 12, pp. 4296-4307, Dec. 2012.

[25] M. Dale, The Algebra of Random Variable. Hoboken, NJ, USA: Wiley, 1979.

[26] P. Mathys and P. Flajolet, " $Q$-ary collision resolution algorithms in random-access systems with free or blocked channel access," IEEE Trans. Inf. Theory, vol. 31, no. 2, pp. 217-243, Mar. 1985.

[27] M. Polese, M. Centenaro, A. Zanella, and M. Zorzi, "M2M massive access in LTE: RACH performance evaluation in a smart city scenario," in Proc. IEEE Int. Conf. Commun., May 2016, pp. 1-6.

[28] N. Marchenko and C. Bettstetter, "Cooperative ARQ with relay selection: An analytical framework using semi-Markov processes," IEEE Trans. Veh. Technol., vol. 63, no. 1, pp. 178-190, Jan. 2014.

[29] A. Laya, L. Alonso, and J. Alonso-Zarate, "Is the random access channel of LTE and LTE-A suitable for M2M communications? a survey of alternatives," IEEE Commun. Surveys Tuts., vol. 16, no. 1, pp. 4-16, First Quarter 2014.

[30] R. A. Howard, Dynamic Probabilistic Systems, Volume II: Semi-Markov and Decision Processes. New York, NY, USA: Dover, 2007.

[31] A. Papoulis and S. U. Pillai, Probability, Random Variables, and Stochastic Processes. Noida, India: Tata McGraw-Hill Education, 2002.

[32] M. Zorzi and R. R. Rao, "On the use of renewal theory in the analysis of ARQ protocols," IEEE Trans. Commun., vol. 44, no. 9, pp. 1077-1081, Sep. 1996.

[33] H. Alves, C. de Lima, P. Nardelli, R. Souza, and M. Latva-aho, "On the secrecy of interference-limited networks under composite fading channels," IEEE Signal Process. Lett., vol. 22, no. 9, pp. 1306-1310, Sep. 2015. 